\title{
Six cases report of differential diagnosis of periapical diseases
}

\author{
Wen-wei Xia, Ya-qin Zhu, Xiao-yi Wang* \\ Department of Endodontics and Operative Dentistry, Shanghai Ninth People's Hospital Affiliated Shanghai Jiao Tong \\ University School of Medicine, Shanghai 200011, China
}

The distinction of some particular forms of periapical area, involving diseases from regular periapical disease, is a matter of considerable importance when choosing a correct treatment. The aim of this study is to describe the differential diagnosis of periapical diseases from six rare cases in clinical practice. The six rare cases are examples of situations where it is difficult to make a differential diagnosis in clinical practice. By retrospective surveys on the clinical examination, radiographs and pathological results, six patients referred to endodontic treatment in our department were analyzed for the accuracy of diagnosis and therapy. The pathoses of the six cases included two atypical radical cysts, periapical cemental dysplasia, cemento-ossifying fibroma, thymus cancer metastasis in the periapical site and tuberculosis. This report indicates that endodontists should be cognizant of a few particular circumstances when clinically treating periapical diseases.

Keywords: periapical diseases; differential diagnosis; endodontic

International Journal of Oral Science (2011) 3: 153-159. doi: 10.4248/IJOS 11055

\section{Introduction}

The periapical disease is one of the most prevalent diseases in general dental practice. However, because the clinical diagnosis and treatment of periapical diseases is quite straightforward, there are few descriptions on differential diagnosis of periapical diseases in either textbooks or reference books. More attention has been paid to periapical diseases, such as periapical abscesses [1], periapical granuloma, and periapical cysts [2]. In practice, a patient who has problems similar to periapical diseases, such as radical cysts, cemento-osseous dysplasia, or tumors, will also be presented to an endodontist for treatment. Moreover, it has been suggested that the associated symptoms of maxillofacial cancer [3] as well as some systemic diseases such as tuberculosis [4] may

*Correspondence: Xiao-yi Wang

Tel: 8621 23271699; Fax: 862163135412

E-mail: xwwall@sina.com

Received 27 December 2010; Accepted 13 January 2011 appear initially as periapical signs. Thus, early correct diagnosis of such patients was crucial for treatment and subsequent prevention of advanced pathological process.

The aim of this study is to describe the differential diagnosis on periapical diseases based on six rare cases. We hope the cases presented here may be helpful in avoiding the risk of initial endodontic treatment in patients who do not have a periapical pathosis origin.

\section{Case Reports}

The six cases reported in this study are from patients who were diagnosed between 2000 and 2004 at the Department of Endodontics and Operative Dentistry, Shanghai Ninth People's Hospital Affiliated Shanghai Jiao Tong University School of Medicine.

\section{Case 1}

A 72-year-old female patient experiencing toothache with right lower gum swelling for 3 weeks attended the endodontic clinic. The pathological diagnosis was "inf- 
lammation" by biopsy in another hospital before the patient was referred to our department. On clinical examination, it was observed that the bone of teeth 44 and 45 expanded to buccal side without loosing teeth or knocking pain. The tooth 46 was a residual crown. Radiographic examination showed periapical radiolucence of 44 and 45 without obvious white osseous response lines. The alveolar bone resorption of tooth 44 almost reached the apex and tooth 45 reached 1/2 length of the root (Figure 1A). The pulp did not respond
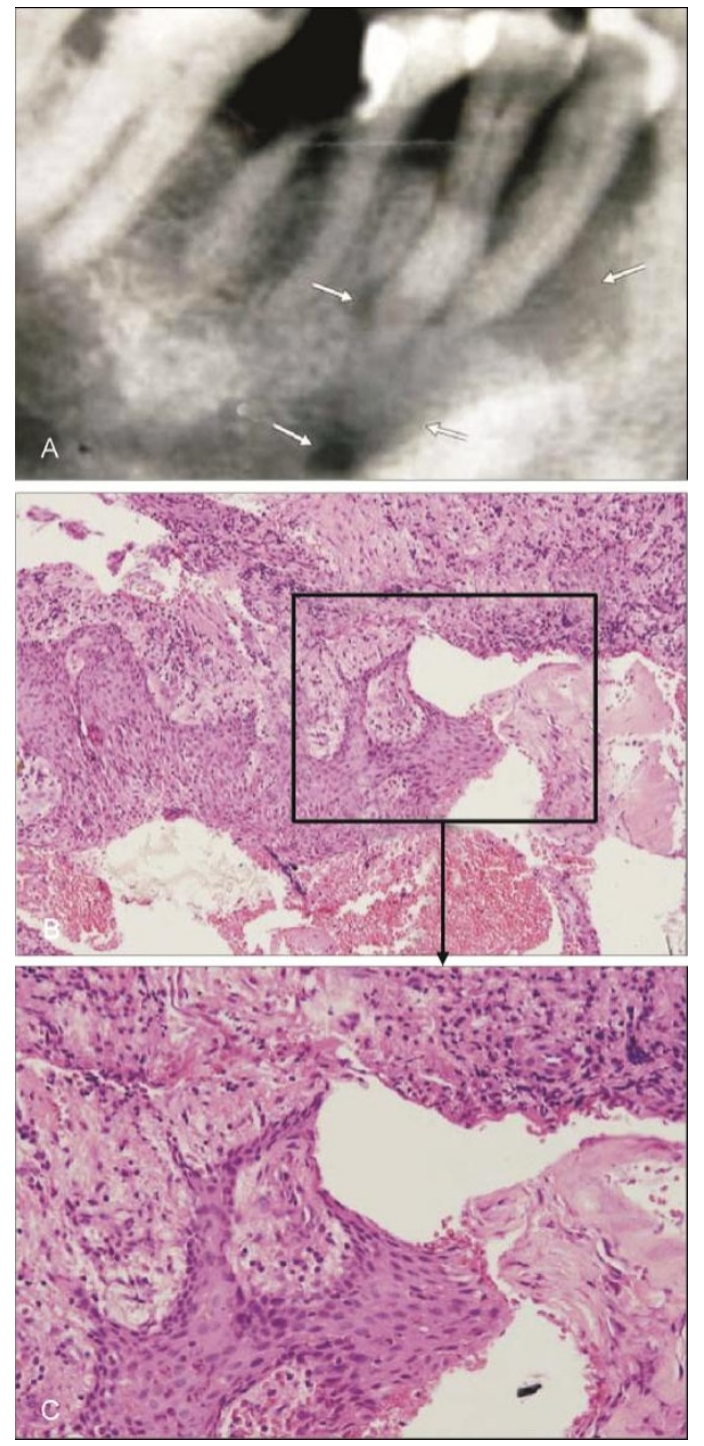

Figure 1 A typical radical cyst. (A) Radiographic image of case 1. Arrows indicate that the shadow of bone absorption reached mental foramen. (B) Histotolgical examination of the biopsy. H\&E stainning, $\times 200$. (C) Histotolgical examination of the biopsy. H\&E stainning, $\times 400$. to electric vital test. The patient therefore diagnosed was referred to Department of Oral \& Maxillofacial Surgery in our hospital to extract teeth $46,45,44$. Upon the surgery, large amount of granulation tissue was resected from the periapical area and a biopsy was taken. The wound healed smoothly 3 weeks post-surgery. The pathological examination showed atypical radical cyst with reactive osseous proliferation (Figure 1B).

\section{Case 2}

A 54-year-old female patient suffering from left upper lip swelling without relief by local anti-inflammatory therapy in other hospitals was referred to our department. On clinical examination, it was observed that the labial gingival and mucosal tissues above the left maxillary lateral incisor were swelling with undulation feeling in palpation. This tooth was intact without caries and responded to the vital pulp test. The labial abscess of tooth 22 was incised and the drainage was kept for 3 days. After the acute inflammation subsided, root canal therapy was performed and the pulp was observed to be intact. Radiographic examination showed a $1 \mathrm{~cm} \times 1.5 \mathrm{~cm}$ radiolucent lesion in the apical area of 22 with smooth, corticated borders indicating a cyst (Figure 2A). The cyst was removed 3 days after the root canal being filled. During the surgery, a small perforation of palatal osseous lamella was observed and most of the granulation tissue was in the palatal side of tooth 22 . At 3 months postsurgery, X-ray examination showed that the destruction of the apical area had been recovered together with the formation of new tissue (Figure 2B). Histopathological examination of the biopsy showed a structure of typical radical cyst (Figure 2C).

\section{Case 3}

A 71-year-old male patient experienced intermittent pain and swelling in right lower molars for more than 2 years. Since root canal therapy was not effective, the tooth was extracted one year prior. However, the extraction inhibited the healing and relief of pain. The patient was then referred to our department and a radiographic examination was performed. Radiographic images showed a high density lesion in either right or left molar areas. A diagnosis of periapical cemental dysplasia of bilateral molars was then made (Figure 3A). The patient was then referred to Department of Oral \& Maxillofacial Surgery. During the surgery, teeth 46, 44, 24, 25 were extracted and it was found that roots of these teeth were grossly enlarged and ossified. Histologically, maxilla and mandible cemental proliferation in bilateral periapical areas was observed (Figure 3B). Thus, final diagnosis was made as multiple periapical cemental dysplasia. 


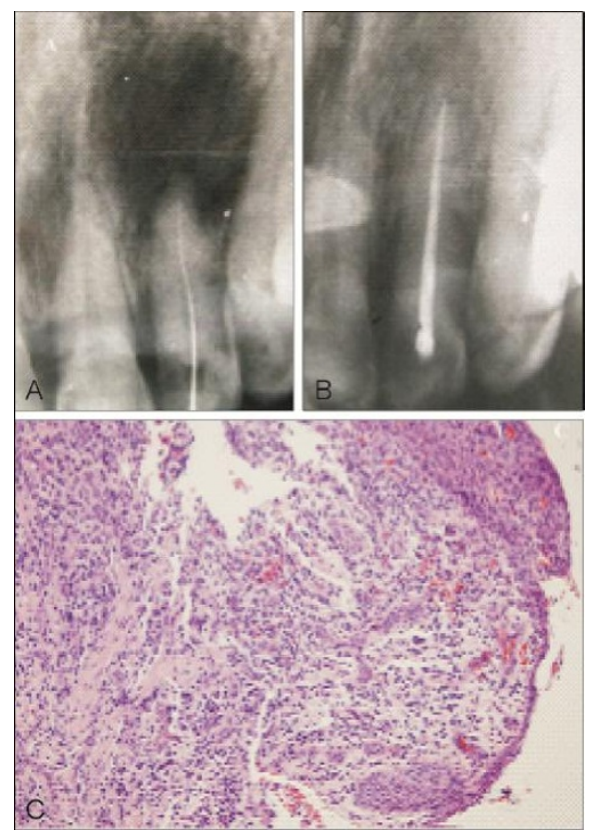

Figure 2 Typical radical cyst. (A) 22 Cyst of the incisive canal $(1.0 \mathrm{~cm} \times 1.5 \mathrm{~cm}$ radiolucence lesion in the apical area of 22 ). (B) 22 Cyst of the incisive canal (The destruction of the apical area recovered after 3 months). (C) Histology observation of biopsy tissue in surgery. Staining, $\times 200$.
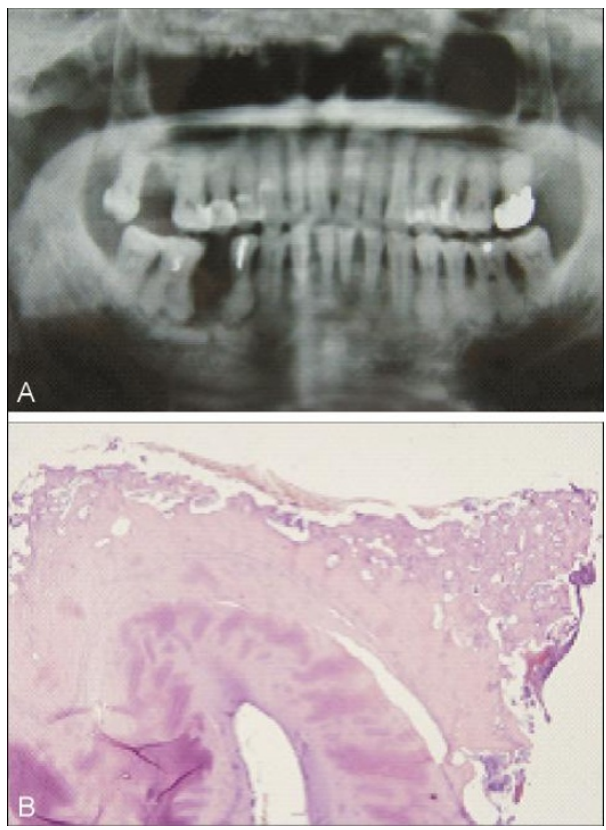

Figure 3 Multiple periapical cemental dysplasis. (A) Radiogram showed high dense radiopacity in bilateral molars. (B) Cemental proliferation in bilateral periapical areas was detected. H\&E staining, $\times 200$.

\section{Case 4}

A 50-year-old female patient experiencing discomfort in the 36 area was referred to our department. Upon examination, a fixed cantilever bridge was found in teeth $36,37,38$. No obvious pain on percussion was observed on tooth 36. Radiographic examination showed a $1.5 \mathrm{~cm} \times 1.5 \mathrm{~cm}$ lesion images at the apical area of the left side molar and a small isolated bone was observed in the center of the lesion. In consideration of the fact that the lesion was near to the apex of 36 , a diagnosis of apical periodontitis with local bone necrosis was made (Figure 4A). The cantilever bridge was removed and the pulp was still alive. The tooth 36 was treated by root canal therapy and a biopsy was taken. Histopathological examination revealed a typical cemento-ossifying fibroma (Figure 4B).

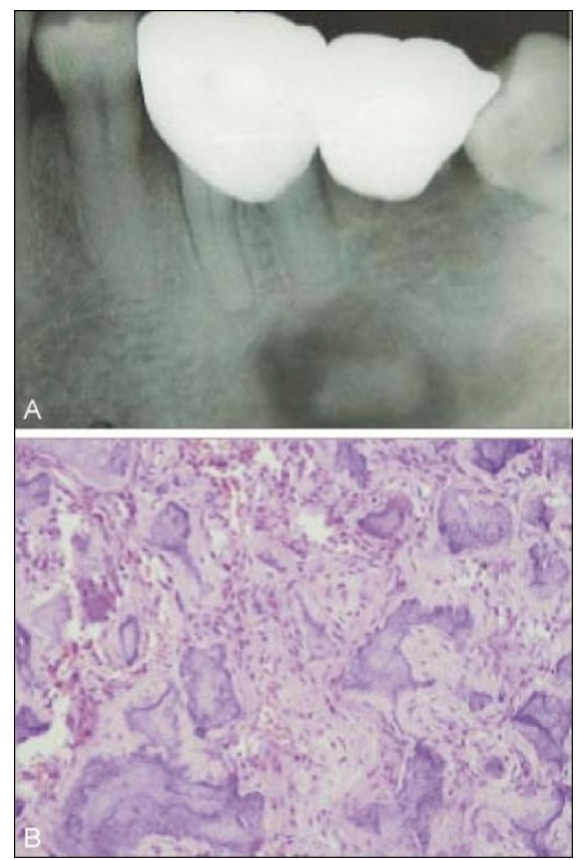

Figure 4 Cemento-ossifying fibroma. (A) Radiogram showed $1.5 \mathrm{~cm} \times 1.5 \mathrm{~cm}$ lesion at the apical area of the left molar. (B) Histology examination of cementoossifying fibroma. H\&E staining, $\times 200$.

\section{Case 5}

A 32-year-old female patient experiencing tooth 47 pain unrelieved by root canal therapy, was referred to our department. On clinical examination, it was found that 47 had pain on percussion and displayed slight local swelling of buccal side. Radiographic examination showed a sparse distribution of bone trabecula, which was speculated as a result of bone resorption, at apical area of 47 , 
48. The tooth of 48 was impacted (Figure 5A). When closely questioned the patient's medical history, it was discovered that she had received a thymusectomy because of the thymus cancer one year ago. Therefore, a suspicious diagnosis of cancer metatasis was made and the patient was referred to emission computed tomograpy (ECT) examination. Images of ECT examination showed exceptionally high density radioactivity in the right mandible, left anterior superior iliac spine, and left hip joint, indicating tumor metastasis (Figures 5B, 5C). The patient then received chemotherapy.

\section{Case 6}

A 48-year-old female patient experiencing localized swelling and pain in the right lower molar area for 2 months, diagnosed with apical periodontitis in other hospitals, was referred to our department. On examination, the gums of teeth 47 and 43 were swolled, 45,44 were lost, and tooth 46 was a residual crown with inflammatory granulation tissue in the mesial gingiva. The patient received anti-inflammation therapy in our department for one month but the swelling and toothache was not relieved. Thus, a biopsy was taken from the mesial side of tooth 46 at which inflammation resulting from tuberculosis was indicated. Radiographic examination showed obvious bone resorption at the furcation of tooth 46 (Figure 6A). Through further clinical examination, we found that the bilateral submandibular lymph nodes were tumescent with the quality of medium to hard. To make a definitive diagnosis, a biopsy was
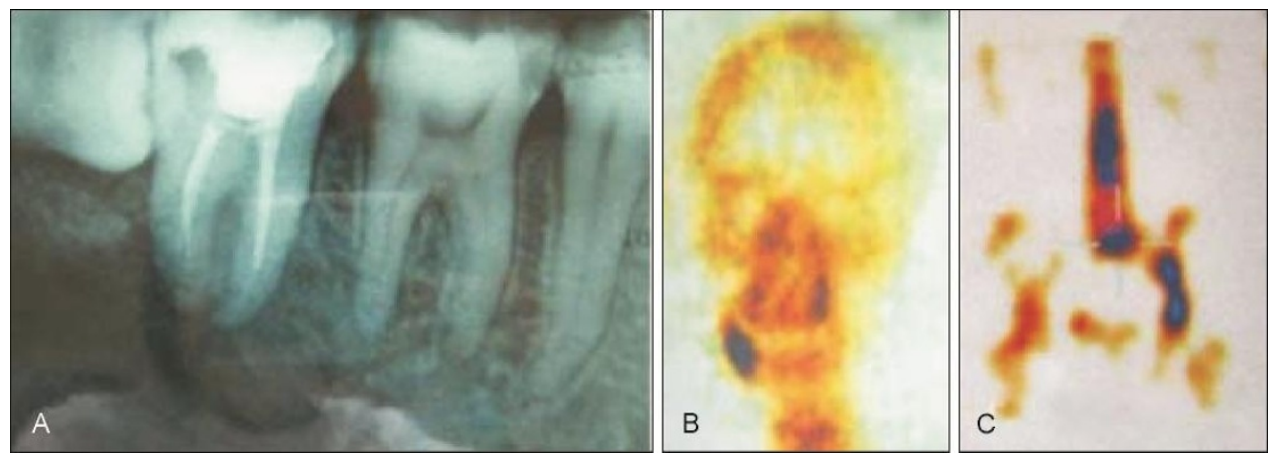

Figure 5 Thymus cancer metastasis. (A) Bone absorption characterized by sparse distribution of bone trabecula at apical area of 47, 48. (B) ECT images of cancer metastasis in right mandible (C) as well as in left anterior iliac and hip joint.
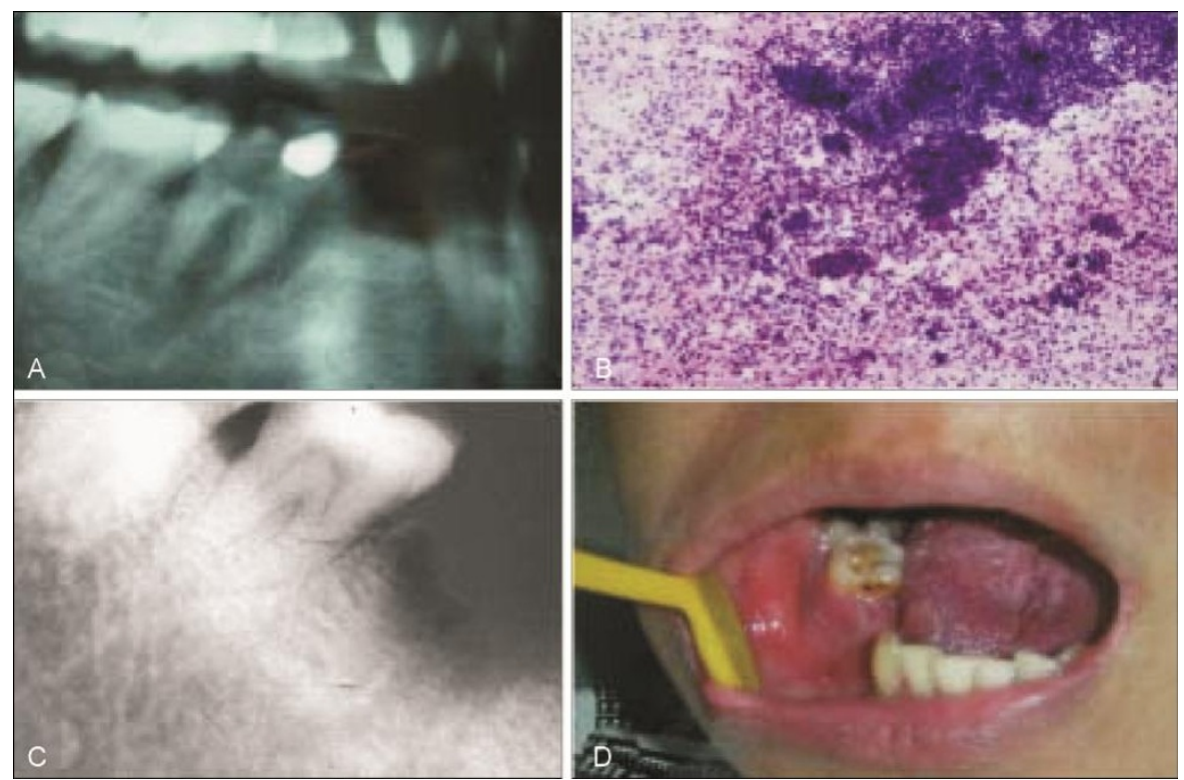

Figure 6 The jaw bone tuberculosis. (A) X-ray images showed bone absorption at furcation of tooth 46. (B) Histology examination displayed tuberculosis. (C) After 4 months of anti-tuberculosis treatment, radiograms showed regeneration of bone (D) as well as healing of the wound resulted from tooth extraction. 
collected from the right submandibular lymph node by aspiration. According to the histopathological examination, distribution of epithelial cells, multinuclear giant cells and lymphocytes were observed, indicating the strong possibility of tuberculosis (Figure 6B). Therefore, anti-tuberculosis treatment was carried out. After 3 months of treatment, the size of tumescent submandibular lymph node reduced remarkably. The granulation tissue in mesial gum and the swelling vanished. In the process of anti-tuberculosis treatment, 46 remnant coronal was broken off and only the remnant root was left. The radiographic image showed that the apical bone was repaired. 46 remnant root was then extracted and the anti-tuberculosis treatment was continued. After 4 months, a follow-up visit was made. The extraction wound was well healed, and the bone was well repaired (Figures 6C, 6D).

\section{Discussion}

The diagnosis of periapical lesions of different origin remains a crucial issue for endodontists because the correct diagnosis has a decisive influence on the subsequent choice of clinical interventional approaches and the resulting success of treatment. However, in some circumstances, it is difficult to identify the periapical diseases from other periapical area-related diseases derived either systemically or locally. Therefore, clear differential diagnosis of periapical lesions is also fundamental for successful treatment of patients experiencing periapical lesions.

The diagnosis of radical cysts mainly depends on radiological examinations. The circular radiolucencies' shadow with clear boundary in the periapex of the tooth, sometimes with the white osseous response line peripherally, remains the classical diagnostic standard for radical cyst. However, the identification of radical cysts, especially in cases which the radiographic images of apical area appeared atypically, can not be made merely by radiological examination. The main difference between atypical radical cysts and typical radical cysst is that there is no radiopaque line at the apical lesion and the boundary is fuzzy on X-ray film. However, the clinical signs of atypical cysts are similar to that of typical cysts. Therefore, in differential diagnosis, clinical signs and $\mathrm{X}$-ray should be taken into consideration together.

The distinction of radical cysts from tumor may be confusing if the diagnosis was made only on radiographic examination. In addition, the correct diagnosis was still difficult even integrating with clinical examination. The periapical radiographic image of case 1 showed irregular, burrow-like bone absorption without obvious osseous response line near the mental foramen. Clinical examination indicated: the teeth 45 and 44 were not obviously loose; there was no pain on percussion; the bone was expended on buccal side. Thus, endodontists may make a diagnosis of tumor. However, the result of pathology of the biopsy demonstrated that it was a radical cyst with reactive osseous proliferation. The massive granulation tissue seen in the surgery indicated that the long lasting heavy inflammation reaction may be responsible for the non-typical X-ray image which caused endodontists to make an incorrect diagnosis of tumor.

The identification of radical cysts from other odontogenic cysts and non-odontogenic cysts, which were classified as jaw cysts, remains another challenge to endodontists. Jaw cysts includes odontogenic cysst, nonodontogenic cysts (such as globlo-maxillary cysts, nasopalatine duct cysst, median cysts, nasolabial cysts), extravasation cysts, and traumatic bone cysts. Among non-odontogenic cysts, nasolabial cysts and cysts of the incisive canal (also named nasopalatine duct cysts) are more easily confused with radical cysts. Anatomical localization and pulp vigor are main points for differential diagnosis of odontogenic cyst and non-odontogenic cysts.

The diagnosis of radical cysts for the case 2 is untenable because the pulp was alive. The diagnosis of globlo-maxillary cyst also is excluded for this case because X-ray showed the cyst was at the apical area of tooth 22 but not between teeth 22 and 23. Thus, the final diagnosis should be a cyst of the incisive canal. The reason is that incisive hole often appears as a boundary clear circular or elliptic lower density image on X-ray images. Anatomically, the incisive canal originates from lower incisive foramen and stops at upper incisive hole bilaterally. The positions of the incisive canal lower foramen are between the incisors, and near alveolar crest or at the apical level which can be seen on radiograph at incisor apical area. The incisive canal upper foramen located at the front of nose and the bottom of lip, near nasal septum. On the radiographic image of apical area, the incisive canal upper foramen could project to any incisor's apical area. Thus, sometimes it is recognized as periapical disease by mistake.

The main distinction between apical periodontitis and periapical cemental dysplasia is that the necrosis in former is caused by pulpitis, while the latter is caused by a primarily pathological process of cementum [5]. By radiographic examination, the clinical process of the periapical cemental dysplasia was characterized by three different periods including normal alveolus, bone fibrosis and density, and atypical remineralization of bone. However, clinically, the pulp is always alive in any of 
the three circumstances mentioned above and the mineralization of the apical area could be cementum, bone, or mixture of both. The pathological change in periapical cemental dysplasia would cause cortex expansion, mucosal ulcer, secondary infection, even reverse pulpitis and necrosis probably.

The failed distinction of apical periodontitis from periapical cemental dysplasia was responsible for the inefficient relief of toothache of patient in case 3 who had an unnecessary root canal therapy experience. In fact, all three different processes of periapical cemental dysplasia, but mainly the mature stage, were observed by radiology in case 3 of this study. More importantly, the pulp in this case is alive. The reason why a correct differential diagnosis in this case was not made lies on the fact that the diagosis was based mainly on the periapical X-ray image. Thus, pantomography is the key for diagnosis of this case.

Cemento-ossifying fibroma, which was once called "cementifying fibroma" and classified as a subtype of cementoma, is the pathological change of fibro-osseous tissues. As the cementum is histologically similar to the texture of bone tissue, it is very difficult to distinguish the cemento-ossifying fibroma from ossifying fibroma pathologically. Therefore, the classification of these two diseases had been listed as one which named as Cementoossifying fibroma according to the WHO revised classification since the year of 1992 [6].

The morbility of cemento-ossifying fibroma is rare in clinical practice. Most of cemento-ossifying fibroma occurs in the root of a tooth, forming not only bone trabecula but also cemental tissue. Thus, it is also classified as an odontogenic tumor. In most circumstances, cemento-ossifying fibroma is easily confused with periapical diseases at the early stage when the pathological lesion is limited within the jaw bone.

The basic manifestation of cemento-ossifying fibroma in radiographic examination includes multiple cysts, ossification and osteopenia. However, according to our experience, the key point of differential diagnosis of cemento-ossifying fibroma with apical periodontitis is that the pulp is alive in cemento-ossifying fibroma but not in apical periodontitis. This is consistent with what had happened in the patient of case 4 . In this patient, the chronic apical periodentitis was wrong diagnosed by the fact that tooth 36 had received crown repair without root canal therapy in other hospital. On the basis of this diagnosis, the small calcified shadow with bone-like density in X-ray images was neglected and suspected as the dead bone remnant caused by inflammation. However, the diagnosis of apical periodontitis was denied and a diagnosis of cemento-ossifying frbroma was made in our department.

Tumorigenesis of jaw bone is usually the result of metastasis of cancer originally derived from breast [7], lung [8] as well as prostate [9], as previously reported. To our knowledge, no report has been found about the jaw metastasis of cancer originating from thymus, which is extremely rare in clinical practice. Unawareness of the thymus cancer metastasis as well as an insufficiently detailed medical history inquiry may be responsible for the misdiagnosis of periapical endodontitis and subsequent nonessential root canal therapy in this patient of case 5 .

Oral tuberculosis which is not a common disease in clinic may be located in soft tissue of oral cavity, or osseous-tissue in the jaw. Symptoms of oral soft tissue tuberculosis may typically be shown as latent mucosa ulcer and severe pain [4].

However, it is interesting to notice that the oral tuberculosis without pain occurred in the patient of case 6. Together with the observation of nonvital of 46 , a misdiagnosis of periapical disease was made and a subsequent non-effective periapical therapy was carried out for 2 months before the patient was referred to our department. A final correct diagnosis of tuberculosis was made based on the histopathologic examination in combination with ineffective anti-inflammation therapy. Therefore, a proper diagnosis of periapical disease should not merely be based on examination of tooth and alveolous area, while comprehensive examination of the whole oral cavity and maxillofacial issues was also important.

In conclusion, the diagnosis for periapical diseases in general is quite straightforward. However, in some peculiar circumstances, endodontists should be cautious to avoid the confusion of the apical diseases with other periapical-related diseases in clinical practice. The key point of differential diagnosis is that the pulps of periapical diseases are usually not viable, whereas the pulp is often alive in apical area related jaw diseases and system disease. Meanwhile, the history of system disease and clinical signs may also exist. Hence, prior to initiating any treatment, vital pulp test, a comprehensive examination in oral cavity as well as maxillofacial area not only limited to apical site, and when necessary, localized radiographic examination, pantomography, even $\mathrm{CT}$ or other general physical examination should all be taken into consideration.

\section{Acknowledgements}

We thank Pro. Wei-liu Qiu for his revision of this manuscript and Dr. Li-zhen Wang for her help in histological examination. This study was supported by 
Science and Technology committee of Shanghai (10JC 1408800).

\section{Reference}

1 Huang $\mathrm{CH}$, Brunsvold MA. Maxillary sinusitis and periapical abscess following periodontal therapy: a case report using three-dimensional evaluation. $J$ Periodontol 2006; 77: 129-134.

2 Rózyło-Kalinowska I. Digital radiography density measurements in differentiation between periapical granulomas and radicular cysts. Med Sci Monit 2007; 13: 129-136.

3 Martins MD, Taghloubi SA, Bussadori SK, et al. Intraosseous schwannoma mimicking a periapical lesion on the adjacent tooth: case report. Int Endod J 2007; 40: 72-78.

4 Ertas U, Tozoglu S, Uyanik MH. Submandibular tuberculous lymphadenitis after endodontic treatment of the mandibular first premolar tooth: report of a case. J Endod
2006; 32: 1107-1109.

5 Dunfee BL, Sakai O, Pistey R, Gohel A. Radiologic and pathologic characteristics of benign and malignant lesions of the mandible. Radiographics 2006; 26: 1751-1768.

6 Kramer IR, Pindborg JJ, Shear M. The World Health Organization histological typing of odontogenic tumours. Introducing the second edition. Eur J Cancer B Oral Oncol 1993; 29B: 169-171.

7 Dib LL, Soares AL, Sandoval RL, Nannmark U. Breast metastasis around dental implants: a case report. Clin Implant Dent Relat Res 2007; 9: 112-115.

8 Park JY, Kim HS, Zo JI. Initial presentation of lung sarcomatoid carcinoma as a metastatic lesion in the mandibular gingiva. $J$ Periodontol 2006; 77: 734-737.

9 Reyes Court D, Encina S, Levy I. Prostatic adenocarcinoma with mandibular metastatic lesion: case report. Med Oral Patol Oral Cir Bucal 2007; 12: E424-E427. 